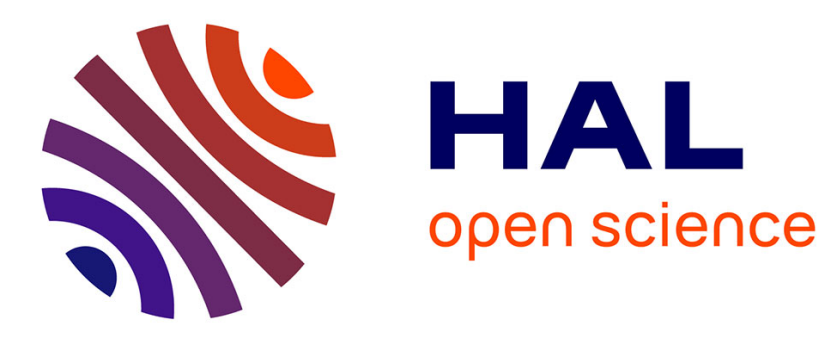

\title{
Quand Anouilh entre dans la danse
}

\author{
Hélène Laplace-Claverie
}

\section{To cite this version:}

Hélène Laplace-Claverie. Quand Anouilh entre dans la danse. Revue d'histoire littéraire de la France, 2010, 110 (4), pp.863-871. hal-02171764

\section{HAL Id: hal-02171764 https: / hal-univ-pau.archives-ouvertes.fr/hal-02171764}

Submitted on 11 Feb 2020

HAL is a multi-disciplinary open access archive for the deposit and dissemination of scientific research documents, whether they are published or not. The documents may come from teaching and research institutions in France or abroad, or from public or private research centers.
L'archive ouverte pluridisciplinaire HAL, est destinée au dépôt et à la diffusion de documents scientifiques de niveau recherche, publiés ou non, émanant des établissements d'enseignement et de recherche français ou étrangers, des laboratoires publics ou privés. 


\section{Quand Anouilh entre dans la danse}

Hélène Laplace-Claverie

Université d'Avignon

Il y a dans le théâtre de Jean Anouilh, en particulier dans son premier théâtre, quelque chose comme une qualité dansante. Du Bal des voleurs (1938) à Ornifle (1955) en passant par Léocadia (1940) et autres Pièces Roses ou Brillantes, le recours à la musique n'est qu'un des signes — et peut-être pas le plus intéressant — d'une sorte de tentation chorégraphique souterrainement à l'œuvre au cœur du travail dramaturgique d'Anouilh. On pourrait, mutatis mutandis, appliquer à l'auteur du Voyageur sans bagage ce que Charles Mazouer dit de son maître en comédie :

[...] Molière a introduit dans son théâtre un souci de la cadence qui rappelle et postule la chorégraphie. La pulsion rythmique qui anime ce théâtre fait évidemment davantage penser à la danse qu'à la musique. ${ }^{1}$

Après avoir tenté, dans un précédent $\operatorname{article}^{2}$, de montrer ce que Le Bal des voleurs devait à la tradition moliéresque de la comédie-ballet, je souhaiterais, dans les lignes qui suivent, rendre compte de l'attirance effective d'Anouilh pour la danse scénique, et donner un rapide aperçu des fruits de cette attirance.

Anouilh librettiste de ballet : cet élément, certes mineur, d'une carrière bien remplie est en général passé sous silence par les travaux qui cherchent à donner une vision panoramique de ladite carrière. On notera par ailleurs que la récente «pléiadisation» du théâtre d'Anouilh s'est faite au détriment de textes sans doute jugés trop marginaux, ou trop peu littéraires, pour prendre place aux côtés d'Antigone ou de L'Alouette. Petite parenthèse : il y aurait, pour qui s'intéresse au destin de ces mal aimés de l'histoire éditoriale que sont les arguments chorégraphiques, une très instructive étude comparative à mener. Il s'agirait de feuilleter le Théâtre complet de quelques dramaturges majeurs du $\mathrm{XX}^{\mathrm{e}}$ siècle, et d'observer si la danse a droit de cité à l'intérieur de ces ouvrages. On découvrirait alors, pour ne prendre que ces exemples, que si la «Pléiade Cocteau » offre une place de choix à Parade et aux Mariés de la tour Eiffel, que si L'Homme et son désir figure dans le théâtre de Claudel et 'adame Miroir dans celui de Genet, en revanche aucun des textes à vocation chorégraphique de Ionesco (Le Jeune homme à marier et Apprendre à marcher) ne semble avoir été jugé digne de rejoindre Les Chaises et Rhinocéros sur papier bible. Loin de moi l'idée de porter un quelconque jugement sur des choix éditoriaux soumis à divers paramètres et tributaires de règles contraignantes. Tout juste me permettrais-je d'espérer qu'un souci excessif de hiérarchisation, voire d'épuration, ne finisse pas par occulter le goût souvent très vif des grands auteurs pour les petites formes et les petits genres. C'est la compréhension même de cet organisme complexe qu'est l'histoire du théâtre qui en dépend.

Dans le cas d'Anouilh, écrire une comédie-ballet dans les années 1930, puis se mettre à plusieurs reprises, après la Deuxième Guerre mondiale, au service d'un jeune chorégraphe plein d'avenir, ne sont pas des décisions anodines. Il y a là quelque chose qui engage le sens d'une œuvre que l'on a peut-être trop systématiquement tendance à enrôler du côté de l'analyse psychologique et de la maîtrise du verbe. Et si les ballets d'Anouilh permettaient

\footnotetext{
${ }^{1}$ Charles Mazouer, Molière et ses comédies-ballets, Paris, Klincksieck, coll. «Bibliothèque de l'Âge classique », 1993, p. 142 (rééd. Paris, Champion, coll. «Lumière classique », 2006).

2 «Le Bal des voleurs de Jean Anouilh : apothéose ou métamorphose de la comédie-ballet?», à paraître en 2010 dans la revue Études littéraires (Université de Laval, Québec).
} 
d'envisager un peu autrement l'univers d'un dramaturge à la fois très connu et relativement méconnu?

\section{La collaboration avec Roland Petit}

Jean Anouilh aurait-il jamais écrit pour la danse si le hasard n'était venu placer sur sa route l'ambitieux et talentueux chorégraphe qui devait ressusciter la tradition du ballet narratif, à une époque où le triomphe de la danse abstraite semblait avéré ? On peut en douter. Passionné de littérature, Roland Petit a su, tout au long de sa luxuriante carrière, puiser son inspiration auprès d'écrivains aussi différents que Goethe, Mérimée, Lautréamont, Proust, Lampedusa, Prévert ou Cocteau, n'hésitant pas, le cas échéant, à solliciter directement le talent d'auteurs vivants.

C'est dans ce contexte qu'il faut replacer sa rencontre avec Anouilh, en 1948. Roland Petit, à cette date, est loin d'être un inconnu. Formé à la discipline académique au sein de l'école de danse puis du corps de ballet de l'Opéra de Paris, il n'a que vingt ans lorsqu'il quitte ce trop confortable cocon pour voler de ses propres ailes. Après avoir fondé en 1945 les Ballets des Champs-Élysées et créé l'année suivante l'un des chefs-d'œuvre de la chorégraphie du $\mathrm{XX}^{\mathrm{e}}$ siècle, Le Jeune homme et la mort, il choisit, en 1948 précisément, de prendre la tête d'une nouvelle compagnie, les Ballets de Paris, qui se produit pour l'essentiel au Théâtre Marigny. C'est alors, raconte le chorégraphe lui-même, que son chemin croise celui de Jean Anouilh. Dans une interview accordée à Gérard Mannoni, il dit avoir rencontré le dramaturge au «Bar des Théâtres », près de la Comédie des Champs-Elysées où l'on jouait ses pièces. "Je me rappelle, ajoute-t-il, que nous sommes partis aux sports d'hiver avec sa fille et que j'ai eu un accident en faisant de la luge $[\ldots]^{3} \gg$. Ce récit est (presque) corroboré par un article paru dans Paris-Presse le 2 avril 1948 : " Cet hiver, Jean Anouilh et moi avons fait du ski ensemble à Morzine [...] Un accident "bénin !" nous immobilisa en même temps. C'est ainsi que naquirent Les Demoiselles de la nuit. " Ski ou luge, au final qu'importe. On retiendra que c'est sans préméditation, de façon fortuite, qu'Anouilh devint librettiste de ballet.

Parmi les trois scénarios qu'il conçut pour Roland Petit, deux portent effectivement sa signature et méritent de retenir l'attention. Le troisième, concrétisé tardivement en 1989, est une adaptation du Diable amoureux de Cazotte, que le chorégraphe aurait conservée dans ses tiroirs pendant plus de vingt ans avant de franchir le pas de la traduction scénique ${ }^{4}$. Je ne prendrai pas en compte ce dernier spectacle, pour lequel la contribution d'Anouilh semble se limiter à l'impulsion initiale.

Mais revenons à la genèse des Demoiselles de la nuit, en 1948. "Anouilh avait une connaissance du théâtre proprement miraculeuse, confie Roland Petit à propos de cette œuvre. Son découpage était parfait. Je n'avais plus qu'à m'y glisser. ${ }^{5}$ » À l'évidence, c'est pour son savoir-faire de dramaturge qu'Anouilh fut sollicité par Petit. En véritable émule des Ballets russes, ce dernier a toujours su non seulement s'entourer, mais tirer le meilleur parti de chaque «corps de métier ». Combien de musiciens, de peintres, d'écrivains, de couturiers et bien sûr de danseurs lui ont fourni, au fil des ans, les matériaux nécessaires à son travail de création ? Sauguet, Auric, Messiaen, Ernst, Picasso, Brassaï, Simenon, Saint-Laurent,

\footnotetext{
${ }^{3}$ Gérard Mannoni, Roland Petit : un chorégraphe et ses peintres, Paris, Hatier, 1990, p. 63.

${ }^{4}$ Sans doute le charme androgyne de la ballerine italienne Alessandra Ferri n'est-il pas pour rien dans cette décision tardive de Roland Petit, chorégraphe toujours soucieux de trouver l'interprète idéal de chaque personnage. Une captation vidéo du Diable amoureux est disponible en DVD.

${ }^{5}$ Gérard Mannoni, Roland Petit, op. cit., p. 63.
} 
Babilée, Noureev, pour ne citer que quelques exemples, ont associé leur nom à celui de Roland Petit. Dans le cas des Demoiselles de la nuit, c'est Jean Françaix qui fut chargé d'élaborer la partition, Leonor Fini d'imaginer décors et costumes, tandis que la célèbre ballerine britannique Margot Fonteyn conférait son élégance naturelle au personnage d'Agathe, la chatte tiraillée entre sa nature animale et l'impérieux désir de devenir une femme.

Ce texte étant difficilement accessible, voici l'argument complet des Demoiselles, tel qu'il figure dans le programme des Ballets de Paris, saison 1948 :

Une grande maison obscure, et que tout le monde croit déserte, aux environs de la ville. Il faut traverser un grand parc pour y accéder.

Un petit jeune homme, un musicien, y a été convoqué à une heure tardive, pour y faire un cachet. Il a cru comprendre qu'il s'agissait d'une cérémonie de mariage.

Le petit jeune homme, après s'être perdu plusieurs fois, arrive enfin à la villa du baron de Grotius. Cette maison est uniquement habitée par des chats et c'est eux qui l'ont convoqué, car, en effet, ce soir ils célèbrent une noce. Tous les mois, le baron-chat épouse une des petites chattes de son harem. Tout ce monde est sur deux pattes et bien habillé, il parle comme vous et moi — ou enfin, pourrait le faire s'il ne s'agissait d'un ballet — jusqu'à minuit où, le douzième coup sonné, ils redeviennent tous des bêtes.

De toutes les chattes du baron, l'une d'elles, Agathe, celle qu'il épouse justement ce soir, est différente. Elle a beaucoup lu (sans doute le grenier de cette villa abandonnée est-il plein de livres). Elle rêve de l'amour des hommes, de ce qui le distingue de l'amour-chat: la tendresse et mille subtilités sur lesquelles elle se fait beaucoup d'illusions. Dans un livre, elle a même lu que si elle pouvait être aimée un jour d'un vrai homme, malgré sa triste condition, à minuit, à l'heure où chaque soir tous redeviennent chats, elle resterait femme.

Ce miracle se produit en effet. Après bien des péripéties, le petit jeune homme qui l'aime emmène Agathe parmi les hommes tandis que tous les chats miaulant de rage, se sauvent au douzième coup sur les toits.

Leur amour sera difficile. Malgré tout son désir d'être une vraie femme, de bien faire son ménage, de bercer son petit enfant imaginaire, d'être fidèle, Agathe reste une chatte et les chats ses amis viennent l'appeler chaque nuit sur les toits.

Une nuit, n'y tenant plus, elle les suivra, et le petit jeune homme aux pieds trop lourds tombera et mourra en voulant la poursuivre.

Et Agathe qui peut tout au moins lui être fidèle dans la mort — c'est plus facile —, refaisant tendrement tous ses gestes de vraie femme, viendra se coucher près de lui pour mourir.

Créée au Théâtre Marigny le 22 mai 1948, cette pièce à la fois rose et noire a connu un succès honorable (une centaine de représentations en tout). Le ballet est même entré dès 1951 au répertoire de l'American Ballet Theatre. Nous reviendrons plus loin sur le contenu et la facture d'une histoire ancrée dans la double tradition des contes et légendes d'une part, des canevas chorégraphiques de l'autre, mais dans laquelle transparaissent tout aussi évidemment les préoccupations de l'auteur de La Sauvage ou d'Eurydice.

Intéressons-nous pour l'heure au deuxième ballet issu de la collaboration PetitAnouilh, Le Loup, représenté pour la première fois au Théâtre de l'Empire le 17 mars 1953. Les rôles principaux étaient interprétés par Violette Verdy, Claire Sombert, Georges Reich et Roland Petit. Jean Carzou signait les décors. Quant à la partition, elle marquait les premiers pas d'Henri Dutilleux dans le monde de la danse. Absent de Paris depuis plusieurs années pour cause de carrière américaine, Roland Petit souhaitait avec ce ballet reconquérir le public 
français. Souhait pleinement exaucé, puisque Le Loup remporta un immense succès, jamais démenti au fil du temps. Le spectacle a été dansé des centaines de fois dans le monde entier. Entré au répertoire de l'Opéra de Paris en 1975, il est considéré comme l'un des fleurons de la danse figurative et poétique française du $\mathrm{XX}^{\mathrm{e}}$ siècle.

Réécriture de La Belle et la Bête, conte cruel et médiéval, le livret du Loup est officiellement issu d'une collaboration entre Jean Anouilh et Georges Neveux ${ }^{6}$. Or, comme toujours dans ce genre de situation, il est difficile de faire la part des responsabilités effectives entre les deux hommes de lettres. Selon Roland Petit, l'œuvre serait le fruit d'une commande passée à l'auteur de Colombe, lequel aurait emprunté à Georges Neveux l'idée directrice du scénario :

Je suis allé voir mon ami Jean Anouilh avec lequel, en 1948, j'avais déjà créé Les Demoiselles de la nuit au Théâtre Marigny, pour lui demander une fois encore d'imaginer un conte fantastique à danser. Il se souvenait d'une histoire cruelle que lui avait quelques années plus tôt racontée Georges Neveux. Mais ce dernier n'était pas un conteur comparable à Anouilh. Jean savait prendre une simple histoire et en tirer un sujet étourdissant, étonnamment théâtral. ${ }^{7}$

Il est évident que Roland Petit, en fin stratège, a tout intérêt à souligner l'influence du seul Anouilh sur la genèse de son ballet. Reste que le chorégraphe a le mérite d'attirer l'attention sur une facette peu fréquemment mise en exergue du dramaturge : son talent de raconteur - plus que d'inventeur — d'histoires. On songe bien sûr aux très rares incursions d'Anouilh dans le domaine narratif, qu'il s'agisse de la nouvelle fantastique Histoire de M. Mauvette et de la fin du monde (1939) ou du recueil de fables publié en 1962 dont nous reparlerons plus loin.

En termes de plaisir de lecture, on ne peut certes qu'être déçu par l'argument du Loup, tel qu'il figure dans les brochures-programmes accompagnant les reprises successives de l'œuvre. Beaucoup plus lapidaire que celui des Demoiselles, le texte co-signé par Anouilh et Neveux se contente de résumer l'intrigue du ballet de la façon la plus schématique :

Le jour même de son mariage, un jeune homme trop insouciant s'enfuit avec une bohémienne.

Grâce à la complicité d'un montreur d'animaux, il fait croire à la mariée qu'il s'est changé en loup. La mariée s'en va donc au bras de celui qu'elle prend pour son époux.

Mais, peu à peu, elle découvre que ce loup est un vrai loup.

Sa première frayeur passée, elle se sent attirée par cet être qui, à l'inverse des hommes, est incapable de faiblesse et de mensonge.

Aussi, quand les gens du village prévenus donneront la chasse au loup, elle le défendra et mourra avec lui.

En dépit du caractère rudimentaire de ce synopsis, ou peut-être grâce à cette concision même, les linéaments de la fable apparaissent avec netteté. Et de nombreux points communs peuvent dès lors être mis en lumière entre les deux ballets d'Anouilh.

${ }^{6}$ Georges Neveux (1900-1982): journaliste, dramaturge et scénariste qui fut, comme Anouilh, le secrétaire de Louis Jouvet. On lui doit la pièce Juliette ou la clé des songes (1930), portée à l'écran par Marcel Carné en 1951.

${ }^{7}$ Extrait du programme du Ballet de l'Opéra de Paris, saison 1992-1993, p. 40, cité dans Zizi Jeanmaire, Roland Petit : un patrimoine pour la danse (catalogue d'exposition), sous la dir. d'Alexandre Fiette, Somogy, 2007, p. 129. 


\section{Histoires de bêtes}

Anouilh comme Petit aiment les Fables de La Fontaine. Le premier s'amuse à les pasticher et à les moderniser dans un recueil datant de 1962 ; le second porte à la scène dès 1945, à l'aube de sa carrière, l'œuvre du fabuliste. Rien d'étonnant, par conséquent, à ce que les créations que les deux artistes échafaudent ensemble établissent un dialogue entre humanité et animalité. Fondés sur le thème de la métamorphose (que celle-ci soit avérée ou supposée, réelle ou mensongère, véridique ou fictive), les canevas des Demoiselles de la nuit et du Loup jouent tous deux sur l'effet de miroir (déformant) qui existe entre l'homme et la bête. Dans le scénario de 1948, Anouilh revisite le motif de la tentation anthropomorphique, très à la mode dans le théâtre des années 1930, de La Folle du ciel de Lenormand (1936) à Ondine de Giraudoux (1939). Puis, en 1953, il imagine une variation en forme d'épure autour de la figure fantasmatique du loup-garou, qui n'est pas sans faire écho à la pièce homonyme de Vitrac créée en 1940 au Théâtre des Noctambules; Vitrac dont on sait à quel point Anouilh admirait le talent. De la chatte qui désirait être femme au loup plus humain que les hommes, le dramaturge expert des tourments névrotiques passe en revue toute une gamme d'émotions, d'affects et de pulsions inavouables, que l'art du ballet, par son fonctionnement métaphorique, permet d'explorer paradoxalement plus crûment que le théâtre parlé. Inutile de recourir à de complexes outils psychanalytiques pour comprendre ce que symbolise le héros éponyme du Loup, interprété à la création par Roland Petit lui-même. Comme l'a bien mis en évidence un critique, la gestuelle caractéristique du personnage («doigts pointés, pouces contre les tempes, index tendus au-dessus de la tête $\left.{ }^{8} \gg\right)$ donne immédiatement à percevoir la puissante «attraction sexuelle» qui s'exerce sur la jeune mariée et suscite la haine destructrice des villageois. Sans tomber dans la vulgarité ou le didactisme, Roland Petit stylise, suggère, stimule l'imagination des spectateurs.

Parce que le danseur s'exprime avec son corps, sans le truchement de la parole, parce que la musique et les éléments visuels deviennent les principaux vecteurs de la narration ${ }^{9}$, le spectacle chorégraphique offre à l'auteur dramatique un séduisant espace d'expérimentation. Libéré des impératifs du dialogue et des règles rhétoriques, Anouilh explore les ressources du langage averbal. Sans s'éloigner du réalisme qui lui est cher, il peut repousser les limites de l'analyse psychologique et de la peinture de caractères. À l'instar des moralistes du Grand Siècle, Anouilh portraiture souvent ses personnages avec l'acuité d'un naturaliste : tout se passe comme si l'écriture de livrets de ballet lui permettait d'aller au bout de cette logique, en extériorisant ce qu'il y a d'animal en l'être humain.

Ce faisant, il s'inscrit, volontairement ou pas, dans une tradition solidement établie tout au long de l'histoire du genre chorégraphique. Sans remonter plus avant, mentionnons cette Chatte métamorphosée en femme qui fut l'un des ballets à succès de la monarchie de Juillet, et dont Balanchine, Sauguet et Kochno s'inspirèrent peut-être quand ils créèrent en 1927 La Chatte, pour les Ballets russes de Monte-Carlo. Gageons par ailleurs qu'Anouilh et Petit avaient nécessairement connaissance des Animaux modèles, ballet mis en musique par Francis Poulenc pour Serge Lifar en 1942. Et bien sûr du Lac des cygnes, le célébrissime chef-d'œuvre de Marius Petipa, dans lequel une princesse métamorphosée en oiseau attend,

\footnotetext{
${ }^{8}$ Article de Dominique Frétard paru dans Le Monde, le 18 mai 1993, à l'occasion d'une mémorable reprise du Loup à l'Opéra de Paris.

9 «La partition du Loup est une musique qui "raconte", écrite dans un style volontairement expressionniste, une musique qui veut traduire la poésie des métamorphoses, comme celle des maléfices et de la peur [...] » (Henri Dutilleux, programme du Théâtre national de l'Opéra, 18 mars 1975).
} 
comme l'héroïne des Demoiselles de la nuit, que l'amour d'un homme lui redonne apparence humaine.

Mais sans doute Anouilh avait-il davantage à l'esprit certaines œuvres théâtrales du premier vingtième siècle, qui posent à la fois philosophiquement et scéniquement le problème de la relation entre humanité et animalité, voire, au-delà, entre le genre humain et tout ce qui n'est pas lui, qu'il s'agisse de L'Oiseau bleu (1908), Chantecler (1910) ou L'Enfant et les sortilèges (1925). Comment ne pas penser à cette dernière œuvre, quand on lit dans un compte rendu des Demoiselles de la nuit que le décor représente « un salon 1880 poussiéreux où les meubles ont des pieds de bête et l'horloge un visage de femme ${ }^{10}$ »? Maeterlinck, Rostand, Colette, Ravel... Anouilh : le rapprochement peut paraître iconoclaste, la filiation contestable. Il n'en demeure pas moins que les ballets conçus par l'auteur du Rendez-vous de Senlis ouvrent sur sa production théâtrale une perspective inédite. Ils révèlent une dimension de son imaginaire en général tenue en bride, même si elle transparaît ici ou là au détour de telle pièce, de tel personnage, de tel quiproquo. Certains commentateurs ne s'y sont pas trompés, qui ont parfaitement senti qu'Anouilh librettiste, loin de trahir Anouilh dramaturge, réalisait quelques-unes de ses aspirations profondes. Ainsi Antoine Goléa, à propos des Demoiselles :

Anouilh a trouvé là une des formes les plus heureuses pour le renouvellement de son thème favori de dramaturge : l'impossibilité, pour les êtres, de «sortir de leur peau » afin de se retrouver, libres, identiques, et voués à leur seul amour, par-dessus et au-delà des barrières sociales qui les enserrent. ${ }^{11}$

Autre point de vue, convergent, d'un journaliste sur le même ballet :

[...] en pénétrant dans ce monde mystérieux de la danse, [...] l'auteur de La Sauvage est demeuré fidèle à son style.

L'argument des Demoiselles de la nuit est typiquement « Anouilh ». ${ }^{12}$

Il y aurait donc, entre Anouilh et l'univers chorégraphique, d'inattendues affinités. Loin de se sentir dépaysé, l'homme de théâtre aurait trouvé dans la forme du ballet un écrin parfaitement adapté à sa « vision tragique » et néanmoins « délibérément ludique du monde et de la vie ${ }^{13}$ ». Au souvenir enchanteur de contes à la saveur d'enfance, Les Demoiselles de la nuit et Le Loup superposent l'amertume de récits crépusculaires. Ces deux histoires d'amour et de mort, de fidélité et de trahison, d'exclusion et de rédemption, proclament l'inéluctable séparation de ceux qui enfreignent les lois de l'altérité. On reconnaît là une des problématiques centrales du premier théâtre d'Anouilh, fondé sur la confrontation entre la soif d'idéal et l'impitoyable leçon du réel.

Mais ce que les Pièces Roses ou Noires donnaient à lire et à entendre par le biais d'intrigues réalistes, Les Demoiselles de la nuit et Le Loup le donnent à voir à travers le prisme de fables nimbées de surnaturel. Le pessimisme d'Anouilh en a-t-il perdu de sa virulence ? Sans doute. Mais l'histoire de la danse s'est quant à elle enrichie de deux ouvres atypiques, pleines de charme et de poésie tragique.

Laissons le dernier mot à Roland Petit, qui écrit dans son livre de souvenirs :

\footnotetext{
${ }^{10}$ Article de Claude Hervin, Paris-Presse, 2 avril 1948.

${ }^{11}$ À Présent, 28 mai 1948.

${ }^{12}$ Paris-Presse, 22 mai 1948.

13 Jacqueline Blancart-Cassou, Jean Anouilh. Les jeux d'un pessimiste, Aix-en-Provence, PUP, coll. «Textuelles-Théâtre », 2007, p. 10.
} 
4 octobre 1987

Jean Anouilh est mort.

Je le revois, ses petits yeux en vrille derrière ses lunettes cerclées de métal argenté, des yeux qui savent tout, qui voient tout, et ce regard pointu qui devient tendre lorsqu'il parle de théâtre. Il me disait un jour que les mauvaises critiques lui étaient indifférentes. C'était faux, cela le contrariait et même l'attristait au point, m'a-t-il avoué plus tard, de ne plus avoir envie d'écrire. Heureusement, il n'en a rien fait, pour le bonheur du public et de tous les comédiens qui le joueront avec délectation. ${ }^{14}$

${ }^{14}$ Roland Petit, J'ai dansé sur les flots, Paris, Grasset, 1993, p. 37. 